\title{
Geometrically nonlinear flexural vibrations of plates: In-plane boundary conditions and some symmetry properties
}

\author{
O. Thomas ${ }^{\mathrm{a}, *}$, S. Bilbao ${ }^{\mathrm{b}}$ \\ ${ }^{a}$ Structural Mechanics and Coupled Systems Laboratory, Cnam, 2 rue Conté, 75003 Paris, France \\ b Acoustics and Fluid Dynamics Group, James Clerk Maxwell Building, University of Edinburgh, Mayfield Road, Edinburgh EH93JZ, UK
}

This study is devoted to the derivation of some properties of the von Ka' rma' $\mathrm{n}$ equations for geometrically nonlinear models of plates, with a boundary of arbitrary shape, for applications to nonlinear vibration and buckling. An intrinsic formulation of the local partial differential equations in terms of the transverse displacement and an Airy stress function as unknowns is provided. Classical homogeneous boundary conditions - with vanishing prescribed forces and displace-ments - are derived in terms of the Airy stress function in the case of a boundary of arbitrary geometry. A special property of this operator, crucial for some energy-conserving numerical schemes and called "triple self-adjointness", is derived in the case of an edge of arbitrary shape. It is shown that this property takes a simple form for some classical boundary conditions, so that the calculations in some practical cases are also simplified. The applications of this work are either semi-analytical methods of solution, using an expansion of the solution onto an eigenmode basis of the associated linear problem, or special energyconserving numerical methods.

\section{Introduction}

Thin plates are widely used as parts of various engineering structures. In some applications, a linear model is not sufficient to capture important aspects of their behavior and nonlinearities have to be introduced, especially when the plate is subjected to displacements of the order of magnitude of the thickness. In the cases of both static and dynamic loading, a realistic model has to include geometrical nonlinearities [1]. Under dynamic conditions, numerous nonlinear phenomena can be observed, including energy transfer between modes as well as chaotic vibrations, that a linear model fails to predict [2]. A nonlinear model is also necessary when the plate is subjected to thermal or longitudinal forces that lead to buckling elastic deformation. Whereas the buckling loads can generally be deduced from a linear analysis, the simulation of the plate postbuckling behavior necessitates the introduction of geometrical nonlinearities into the model [3]. A widely used way of introducing geometrical nonlinearity in plate models was introduced in the static case by von Kármán

\footnotetext{
*Corresponding author.

E-mail address: olivier.thomas@cnam.fr (O. Thomas).
} 
in 1910 [4]. It consists of retaining lower order nonlinear quadratic terms in the expressions for longitudinal strain as a function of transverse displacement. This theory has been extended to dynamic problems of homogeneous linear elastic plates in Ref. [5] and to laminated plates - see e.g. Refs. [1,6]. The same ideas are also used to formulate shell theories. Examples are the Donnell-Mushtari-Vlasov theories for shallow shells [7-11]. Another widely used plate theory that includes geometrical nonlinearities is that of Berger [12], in which the first invariant of the strain tensor is neglected. However, this theory leads to erroneous results for some boundary conditions, especially when the edge is free of loads in the in-plane direction [13-15]. von Kármán and Berger theories usually give correct results for large displacements and moderate rotations, if the transverse displacement remains of the order of magnitude of the thickness of the plate. Other theories include higher order nonlinear terms, and account for large rotations. The interested reader is referred to Ref. [16] for a thorough literature review as well as for precise derivations of those formulations.

The von Kármán equations have been used in a huge number of analytical and semi-analytical studies during the past decades, devoted to a very large spectrum of applications: from the modeling and the analysis of nonlinear vibrations and chaos in plates [17] and in percussive musical instruments [18], to the crumpling of paper sheets [19], along with a huge number of engineering studies, from buckling and dynamics of micro/ nanosystems [20,21] to problems in flow-induced vibration [22]. The interested reader is referred to the following reviews of the literature: [23,24] for studies on plates, [25-27] for shells and to recent textbooks on nonlinear vibrations $[2,16,28]$. The von Kármán theory has been found to be attractive to scientists and engineers probably because it is able to simulate complex nonlinear phenomena with an excellent accuracy as compared to experiments (among others, see Refs. [2,28-30]), whereas the nonlinearities are included in a very simple way - by adding only a single bilinear operator to the classical linear Kirchhoff-Love theory.

Aside from the previous cited studies involving particular applications, the von Kármán theory has been rigorously justified in more mathematical works, through a reduction from the nonlinear three-dimensional equilibrium equations. Asymptotic expansions have been used in Refs. [31-34] and lead to a classification of various plate theories - including the von Kármán model - that are valid for a specific scaling of the applied loads as compared to the thickness of the plate. A recent study [35] proposes a further justification. Even if all these studies are restricted to static cases and particular boundary conditions, they give interesting and rigorous justifications of the core of the von Kármán model and in particular the associated nonlinear strain-displacement law. To the knowledge of the authors, similar studies that include the dynamical terms have not yet been published.

The von Kármán equations can take several forms, depending on the choice of the unknowns. The natural choice would be to write the equations with respect to the three unknown displacements. The three obtained equations include several nonlinear terms, which probably explains why they have been scarcely used in practical analytical works [15]. If the plate is free of an externally distributed in-plane forcing, one can derive an alternate formulation with the transverse displacement $w$ and an Airy stress function $F$ as unknowns. In this case, the problem is written with two equations that take a very compact form, with the nonlinear terms concentrated within a single bilinear operator $L(\circ, \circ)$. This formulation is useful when one is interested in modeling the transverse displacement of the plate at first, all other unknown quantities - the stresses and the in-plane displacements - being obtained in post-processing if necessary, as functions of $w$ and $F$.

This $(w, F)$ formulation has been used in a large number of studies in the past, in majority related to analytical or semi-analytical derivations. However, some energy-conserving numerical schemes recently developed by the second author [36] use this $(w, F)$ formulation to simulate the nonlinear and chaotic response of a plate in large deflection vibrations. In those cases, a difficulty related to the present $(w, F)$ formulation is that the in-plane boundary conditions naturally appear in terms of the membrane forces and the in-plane displacement. The first goal of this article is to revisit those boundary conditions in order to express them in terms of $F$ and $w$ only, in the two classical homogeneous cases of a free edge and an immovable edge. The derivation is conducted with intrinsic notations, so that it can be applied to any coordinate system and thus to plates with an edge of arbitrary shape in the numerical simulations. These in-plane boundary conditions, derived in Section 3.2, have already been derived and used in the literature in the case of a plate with a circular edge (see Refs. [37-40]) but not in the general case.

The second goal of this work is to derive a special symmetry property of the bilinear operator $L(\circ, \circ)$, defined in Ref. [36] as "triple self-adjointness." This property can be useful in some practical cases. Firstly, it 
allows considerable simplification when a solution to the von Kármán nonlinear equations is obtained by an expansion onto eigenfunctions, a common manner of deriving semi-analytical solutions $[2,16,40]$. This latter point will be emphasized in this paper. Secondly, this property has also been found crucial in the above-cited energy-conserving numerical method of solution of the von Kármán equations.

This article is organized as follows: the classical governing equations are first presented in Section 2. Then, the homogeneous boundary conditions are derived in terms of $w$ and $F$ in Section 3. A particular spatial discretization of the partial differential equations by an expansion onto the eigenmode basis of the associated linear problem is proposed in Section 4. Finally, Section 5 is devoted to the derivation of the triple selfadjointness property in the general case and to some simplifications that can be exploited for some particular boundary conditions.

\section{Governing equations}

This section gathers all the important hypotheses and formula that lead to the von Kármán equations that will be written in Section 2.2. All equations are written with the help of intrinsic notation and can be applied to a plate with an edge of arbitrary shape. They are equivalent to those found in many textbooks (see e.g. Refs. $[11,1,6,16])$, often written in a particular set of coordinates associated with a particular edge geometry (rectangular or circular). The interested reader will find in Appendix A some precisions about the notations used in this article and in Appendix $\mathrm{C}$ the application of the intrinsic formulation to particular geometries: a rectangular plate and a circular plate.

The underlying hypotheses that lead to the von Kármán equations formulation of Section 2.2 are recalled here:

(1) A Kirchhoff-Love kinematic, where any normal to the plate mid-surface before deformations remains normal to the deformed mid-surface, is used. The transverse shear stresses are thus neglected.

(2) The normal stress along the transverse direction is neglected.

(3) A von Kármán-like strain-displacement law is used, by neglecting the nonlinear terms of higher order in the Green-Lagrange strain tensor plane part.

(4) The material is linear, homogeneous and isotropic.

(5) The in-plane and rotatory inertia terms are neglected.

(6) The in-plane external forcing is neglected.

\subsection{Preliminary derivations}

The plate in its reference configuration occupies a domain $\Omega$ of a three-dimensional Euclidean space, defined by its mid-surface $\mathscr{S}$, a bounded region of a given infinite plane $\mathscr{P}$ (called the mid-plane), and its thickness $h$ by $\Omega=\mathscr{S} \times[-h / 2 ; h / 2]$. We use the right-handed orthonormal basis $\left(\mathbf{e}_{1}, \mathbf{e}_{2}, \mathbf{e}_{z}\right)$, with $\mathbf{e}_{z}$ chosen normal to the mid-surface $\mathscr{S}$ of the plate so that $\left(\mathbf{e}_{1}, \mathbf{e}_{2}\right)$ is a basis of $\mathscr{P}$. In the following, we shall often separate the in-plane components (along $\mathbf{e}_{1}$ and $\mathbf{e}_{2}$ ) and the out-of-plane components (along $\mathbf{e}_{z}$ ) of vectors and tensors. We shall use the Einstein summation rule, with Greek letter subscripts referring to in-plane components (i.e. $\mathbf{e}_{\alpha}$ means $\mathbf{e}_{1}$ or $\mathbf{e}_{2}$, that is to say $\left.\alpha \in\{1,2\}\right)$.

The location of any point $M$ of the plate is defined by $\mathbf{O M}=\mathbf{x}+z \mathbf{e}_{z}$, where $O$ is a given point of $\mathscr{P}$, $z \in[-h / 2 h / 2]$ and $\mathbf{x} \in \mathscr{P}$ are the transverse and in-plane coordinates, respectively. Under hypothesis (1), the displacement field of the plate during its deformations may be written as

$$
\mathbf{U}(\mathbf{x}, z)=\mathbf{u}(\mathbf{x})-z \boldsymbol{\nabla} w(\mathbf{x})+w(\mathbf{x}) \mathbf{e}_{z},
$$

where $\mathbf{u}$ and $w$ are, respectively, the in-plane and transverse displacements of point $M$ and $\boldsymbol{\nabla} w$ is the plane part of the vector gradient of scalar field $w$. Then, using hypothesis (3), the plane part $\boldsymbol{\varepsilon}$ of the Green-Lagrange strain tensor reads

$$
\boldsymbol{\varepsilon}(\mathbf{x})=\varepsilon_{\alpha \beta} \mathbf{e}_{\alpha} \otimes \mathbf{e}_{\beta}=\boldsymbol{\varepsilon}(\mathbf{x})-z \boldsymbol{\kappa}(\mathbf{x})
$$


with membrane strain tensor $\boldsymbol{\varepsilon}=\varepsilon_{\alpha \beta} \mathbf{e}_{\alpha} \otimes \mathbf{e}_{\beta}$ and curvature tensor $\boldsymbol{\kappa}=\kappa_{\alpha \beta} \mathbf{e}_{\alpha} \otimes \mathbf{e}_{\beta}$ that are functions of $\mathbf{u}$ and $w$ :

$$
\boldsymbol{\varepsilon}=\frac{1}{2}\left(\boldsymbol{\nabla} \mathbf{u}+\boldsymbol{\nabla}^{\mathrm{T}} \mathbf{u}+\boldsymbol{\nabla} w \otimes \boldsymbol{\nabla} w\right), \quad \boldsymbol{\kappa}=\boldsymbol{\nabla} \boldsymbol{\nabla} w .
$$

In the above equations, $\otimes$ denotes the tensor product of two vectors, $\nabla \mathbf{u}$ is the tensor gradient of vector field $\mathbf{u}$ and $\boldsymbol{\nabla}^{\mathrm{T}} \mathbf{u}$ is its transpose.

The material is assumed to be linear, elastic, homogeneous and isotropic (Hyp. (4)), of Young's modulus $E$ and Poisson's ratio v. Under hypotheses (2), all out of plane components of the stress tensor are neglected. Then, by defining the membrane forces and moment's tensor fields with integration of the stress tensor plane part $\sigma$ over the thickness of the plate:

$$
\mathbf{N}=\int_{h / 2}^{h / 2} \boldsymbol{\sigma} \mathrm{d} z, \quad \mathbf{M}=\int_{-h / 2}^{h / 2} z \boldsymbol{\sigma} \mathrm{d} z,
$$

one obtains the constitutive laws:

$$
\begin{gathered}
\mathbf{N}=A[(1-v) \boldsymbol{\varepsilon}+v \operatorname{tr} \boldsymbol{\varepsilon} \mathbf{1}], \\
\mathbf{M}=-D[(1-v) \boldsymbol{\nabla} \boldsymbol{\nabla} w+v \Delta w \mathbf{1}],
\end{gathered}
$$

where $A=E h /\left(1-v^{2}\right)$ and $D=E h^{3} /\left[12\left(1-v^{2}\right)\right]$ are, respectively, the membrane stiffness and the flexural stiffness of the plate.

\subsection{Equations of motion}

By applying a variational formulation (Hamilton's principle or the principle of virtual work) to the plate, one obtains the local equations of motions in terms of $\mathbf{N}$ and $\mathbf{M}$. Then, by neglecting the in-plane and rotatory inertia terms (hypothesis (5)), and in the particular case of vanishing external membrane loading (hypothesis (6)), the in-plane equilibrium equation is satisfied exactly by defining the following Airy stress function $F$ :

$$
\Delta F \mathbf{1}-\nabla \nabla F \equiv \mathbf{N}
$$

In this case, the equations of motions can be written:

$$
\begin{gathered}
D \Delta \Delta w+\rho h \frac{\partial^{2} w}{\partial t^{2}}=L(w, F)+p(\mathbf{x}), \\
\Delta \Delta F=-\frac{E h}{2} L(w, w),
\end{gathered}
$$

where $p(\mathbf{x})$ is a normal pressure loading and $\rho$ denotes the mass density of the material. The interested reader can refer to Ref. [41] or to the textbooks $[15,6,16]$ for exhaustive mathematical derivations. The problem is now defined in terms of two unknown scalar fields - transverse displacement $w$ and Airy stress function $F$ that are solutions of the two scalar equations (7a,b). Eq. (7) is the transverse equation of motion and Eq. (7b) comes from the compatibility condition [1].

The bilinear operator $L(\circ, \circ)$ introduced in the above equations appears in any von Kármán-like plate or shell theory $[1,6-8,10]$. It is sometimes referred to as the "Monge-Ampère form" [34] and will be named in this work as the "von Kármán operator". It is defined by

$$
L\left(w_{1}, w_{2}\right)=\Delta w_{1} \Delta w_{2}-\nabla \nabla w_{1}: \nabla \nabla w_{2},
$$

where : denotes the doubly contracted product of two tensors (see Appendix A). One can remark that all the nonlinear terms of the equations of motion are included within this single operator.

It is worth remarking that the Airy stress function, as defined by Eq. (6) as a function of $\mathbf{N}$ through its second derivatives, is unique up to an arbitrary function, bilinear in the components of $\mathbf{x}$. However, this nonuniqueness is not a problem as $F$ is only an auxiliary function that enables to solve the problem and to obtain the physical variable $w$, and in a second step, $\mathbf{u}$ and $\mathbf{N}$. In a practical problem, $F$ can by uniquely determined either by some particular boundary conditions [31,34] or by adding linear conditions on the boundary that do 

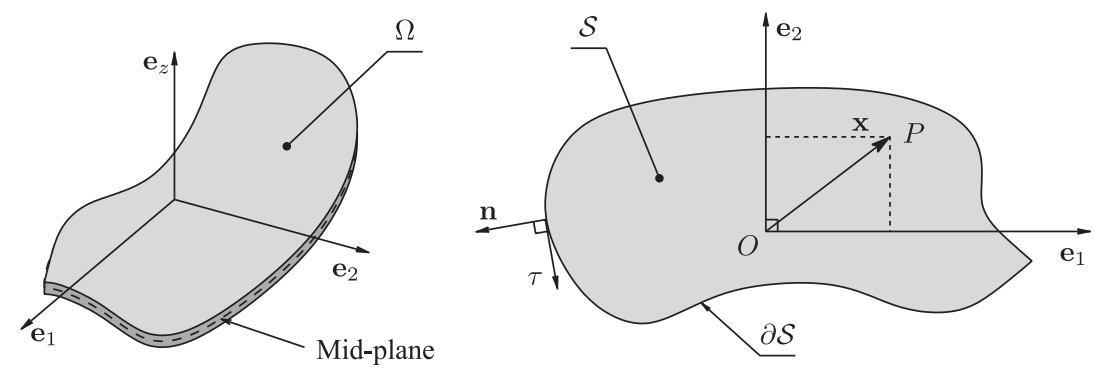

Fig. 1. Sketch of domain $\Omega$ occupied by the plate and its middle plane surface $\mathscr{S}$ with edge $\partial \mathscr{S}$.

not change the value of $w, \mathbf{u}$ and $\mathbf{N}$. The particular discretization procedure described in Section 4 also leads to a unique determination of $F$.

\subsection{Boundary conditions}

The boundary is subjected to an external forcing, represented by a force field $T_{e} \mathbf{e}_{z}+\mathbf{N}_{e}$ and a moment field $\mathbf{M}_{e}$, with $\mathbf{N}_{e}$ and $\mathbf{M}_{e}$ two vectors parallel to the plate mid-plane. One can also impose an in-plane displacement $\mathbf{u}_{e}$, a transverse displacement $w_{e}$ or a normal rotation of the edge $w_{n e}$. The boundary conditions can be obtained in a classical manner using the principle of virtual work or the Hamilton principle $[41,1,6,16]$. At any point of the plate boundary $\partial \mathscr{S}, s$ denotes the arc-length and $(\mathbf{n}, \tau)$ are the normal and tangent unit vector (see Fig. 1). The boundary conditions are:

$$
\begin{gathered}
\mathbf{u}=\mathbf{u}_{e} \quad \text { or } \quad \mathbf{N n}=\mathbf{N}_{e}, \\
w=w_{e} \quad \text { or } \quad Q_{n}+\frac{\partial M_{n \tau}}{\partial s}=T_{e}+\frac{\partial}{\partial s}\left(\mathbf{M}_{e} \cdot \tau\right), \\
\boldsymbol{\nabla} w \cdot \mathbf{n}=w_{n e} \quad \text { or } \quad M_{n n}=\mathbf{M}_{e} \cdot \mathbf{n},
\end{gathered}
$$

where $Q_{n}=(\operatorname{div} \mathbf{M}+\mathbf{N} \nabla w) \cdot \mathbf{n}, M_{n n}=\mathbf{n} \cdot \mathbf{M n}$ is the normal bending moment and $M_{n \tau}=\tau \cdot \mathbf{M n}$ is the normal twisting moment. One can note that considering Eq. (5b) and identities (B.20, B.21, B.22) leads to

$$
\operatorname{div} \mathbf{M}=-D \boldsymbol{\nabla}(\Delta w) \text {. }
$$

In addition to the above boundary conditions (9a-f), the balance with Kirchhoff's corner load has to be fulfilled [42]: at any angular point of coordinate $\tau_{0}$ of the plate boundary $\partial \mathscr{S}$,

$$
w=w_{e} \text { or }\left[M_{n \tau}-\mathbf{M}_{e} \cdot \tau\right]_{\tau_{0}^{-}}^{\tau_{0}^{+}}=0,
$$

where $[f]_{a^{-}}^{a^{+}}=f(a+\varepsilon)-f(a-\varepsilon)$, with $\varepsilon \ll a$, denotes the variation of function $f$ around point $a$.

The purpose of the following sections is to rewrite the boundary conditions $(9 \mathrm{a}-\mathrm{h})$ in terms of unknowns $w$ and $F$ only. We restrict ourselves to the cases of homogeneous boundary conditions, defined by the vanishing of the applied loads $\left(\mathbf{N}_{e}=\mathbf{M}_{e}=T_{e}=0\right.$ on $\left.\partial \mathscr{S}\right)$ and the applied displacements $\left(\mathbf{u}_{e}=w_{e}=w_{n e}=0\right.$ on $\partial \mathscr{S})$.

\section{Boundary conditions in terms of $(w, F)$}

In this section, we use an orthogonal coordinate system to parametrize the plate boundary $\partial \mathscr{S}$, so that $\partial \mathscr{S}$ is exactly a coordinate line. For details about the curvilinear coordinate systems used in this work as well as on differential operators, the reader can refer to Appendix B. $(n, \tau)$ denotes the two curvilinear coordinates and $\mathscr{C}_{n}$ and $\mathscr{C}_{\tau}$ are the corresponding coordinate lines. $\partial \mathscr{S}$ coincides with $\mathscr{C}_{\tau}$ for a particular value of $n . \mathbf{n}$ and $\tau$ still denote unit vectors normal and tangent to $\partial \mathscr{S}$ (see Fig. 1). $R_{n}$ and $R_{\tau}$ are the curvatures of $\mathscr{C}_{n}$ and $\mathscr{C}_{\tau}$ and if $\mathbf{f}$ denotes the mapping that defines the coordinate system, $h_{n}=\|\partial \mathbf{f} / \partial n\|$ and $h_{\tau}=\|\partial \mathbf{f} / \partial \tau\|$, 
so that

$$
\mathbf{n}=\frac{1}{h_{n}} \frac{\partial \mathbf{f}}{\partial n}, \quad \tau=\frac{1}{h_{\tau}} \frac{\partial \mathbf{f}}{\partial \tau} .
$$

The arc-length $s$ on the plate boundary $\partial \mathscr{S}$ is then defined by $\mathrm{d} s=h_{\tau} \mathrm{d} \tau$.

\subsection{Flexural boundary conditions}

To be exhaustive, the classical homogeneous flexural boundary conditions are rewritten here in terms of $w$ and $F$ only.

- In the case of a transversely and rotationally immovable edge, $w_{e}=w_{n e}=0$ and Eqs. (9c, e) are written as

$$
w=0, \quad \boldsymbol{\nabla} w \cdot \mathbf{n}=\frac{\partial w}{\partial n}=0 .
$$

- For a transversely free edge, $\mathbf{T}_{e}=0$ and the twisting moment $\mathbf{M}_{e} \cdot \tau$ has to be a constant along the edge. Eq. (9d) together with Eqs. (10) and (5b) leads to

$$
\boldsymbol{\nabla}(\Delta w) \cdot \mathbf{n}+(1-v) \frac{\partial}{\partial s}[\mathbf{n} \cdot(\nabla \nabla w) \tau]+\mathscr{N}_{w}(F, w)=0,
$$

where

$$
\mathscr{N}_{w}(F, w)=\frac{1}{D}(\mathbf{N n}) \cdot \boldsymbol{\nabla} w
$$

is the nonlinear part of this boundary condition. One can remark that $\mathscr{N}_{w}$ is canceled on the edge if $\mathbf{N n}=\mathbf{0}$, and thus in the case of an edge free of loads in the in-plane direction (see Section 3.2.1).

- For a rotationally free edge, $\mathbf{M}_{e} \cdot \mathbf{n}=0$. With Eq. (9f), one obtains:

$$
M_{n n}=\mathbf{n} \cdot \mathbf{M n}=0,
$$

which gives

$$
v \Delta w+(1-v) \mathbf{n} \cdot(\nabla \nabla w) \mathbf{n}=0 .
$$

\subsection{In-plane boundary conditions}

\subsubsection{Free edge in the in-plane direction}

The case of a free edge in the in-plane direction is defined by the vanishing of applied external in-plane forcing at the plate edge, namely $\mathbf{N}_{e}=\mathbf{0}$. With Eqs. (9b,d), one obtains for all points of $\partial \mathscr{S}$ :

$$
\mathbf{N} \cdot \mathbf{n}=\mathbf{0}
$$

that may be written, with the help of Eq. (6), as

$$
(\Delta F \mathbf{1}-\nabla \nabla F) \cdot \mathbf{n}=\mathbf{0} .
$$

This condition can be written in the above-defined orthogonal curvilinear coordinate system with Eqs. (B.15) and (B.16) as given in Appendix B.1. Eq. (18) is then equivalent to the following two scalar equations that the Airy stress function $F$ has to verify at any point on the boundary:

$$
\begin{gathered}
\frac{\partial^{2} F}{\partial \tau^{2}}+\frac{h_{\tau}^{2}}{R_{\tau} h_{n}} \frac{\partial F}{\partial n}-\frac{1}{h_{\tau}} \frac{\partial h_{\tau}}{\partial \tau} \frac{\partial F}{\partial \tau}=0, \\
\frac{\partial^{2} F}{\partial n \partial \tau}-\frac{h_{\tau}}{R_{n}} \frac{\partial F}{\partial n}-\frac{h_{n}}{R_{\tau}} \frac{\partial F}{\partial \tau}=0 .
\end{gathered}
$$


A much simpler expression for these boundary conditions can now be obtained by considering the following conditions, for any point on the boundary:

$$
F=\frac{\partial F}{\partial n}=0
$$

Since the above relation (21a) is valid at any point on the edge $\partial \mathscr{S}$, it necessarily implies

$$
\frac{\partial F}{\partial \tau}=\frac{\partial^{2} F}{\partial \tau^{2}}=\frac{\partial^{2} F}{\partial \tau \partial n}=0
$$

for any point on the boundary, so that Eqs. (20a, b) and consequently Eq. (18) are verified. As a consequence, Eqs. $(21 \mathrm{a}, \mathrm{b})$ are sufficient conditions to impose a free edge in the in-plane direction. To the knowledge of the authors, this last result has been introduced in the literature only by Ciarlet in Ref. [31] and used in Ref. [40] in the case of a circular plate.

\subsubsection{Immovable edge}

The case of an immovable edge is defined by a vanishing in-plane displacement on the plate boundary, that is to say for all points on $\partial \mathscr{S}($ Eqs. $(9 \mathrm{a}, \mathrm{c}))$ :

$$
\mathbf{u}=u_{n} \mathbf{n}+u_{\tau} \tau=\mathbf{0} .
$$

Expressing the above condition, written using displacements, in terms of the Airy stress function $F$ is less straightforward than in the previous Section 3.2.1. The usual approach is first to integrate the local equation (36) and thus obtain an analytical expression for $F$ as a function of $\mathbf{x}$, then to calculate $\mathbf{N}$ with Eq. (6) in order to obtain $\varepsilon$ with the constitutive relation (5a) and finally to integrate $\varepsilon$ to obtain $\mathbf{u}$ with Eq. (3). It is then possible to apply the boundary conditions (23) in terms of $\mathbf{u}$.

Here, we follow an idea introduced in Ref. [39] that consists in writing two scalar equations with $F$ that are consequences of the boundary conditions written in terms of $\mathbf{u}$. We first write the membrane strain tensor $\boldsymbol{\varepsilon}$ as a function of $u_{t}$ and $u_{n}$, by expressing Eq. (3) in curvilinear coordinates with Eqs. (B.9) and (B.11). We have $\boldsymbol{\varepsilon}=\varepsilon_{n n} \mathbf{n} \otimes \mathbf{n}+\varepsilon_{\tau \tau} \tau \otimes \tau+\varepsilon_{n \tau}(\tau \otimes \mathbf{n}+\mathbf{n} \otimes \tau)$ with

$$
\begin{gathered}
\varepsilon_{n n}=\frac{1}{h_{n}} \frac{\partial u_{n}}{\partial n}-\frac{u_{\tau}}{R_{n}}+\frac{1}{2 h_{n}^{2}}\left(\frac{\partial w}{\partial n}\right)^{2}, \\
\varepsilon_{\tau \tau}=\frac{1}{h_{\tau}} \frac{\partial u_{\tau}}{\partial \tau}+\frac{u_{n}}{R_{\tau}}+\frac{1}{2 h_{\tau}^{2}}\left(\frac{\partial w}{\partial \tau}\right)^{2}, \\
\varepsilon_{n \tau}=\frac{1}{2}\left[\frac{1}{h_{\tau}} \frac{\partial u_{n}}{\partial \tau}+\frac{1}{h_{n}} \frac{\partial u_{\tau}}{\partial n}-\frac{u_{\tau}}{R_{\tau}}+\frac{u_{n}}{R_{n}}+\frac{1}{h_{n} h_{\tau}} \frac{\partial w}{\partial n} \frac{\partial w}{\partial \tau}\right] .
\end{gathered}
$$

A first remark is that since Eq. (23) is valid at any point on the edge $\partial \mathscr{S}$, it necessarily implies that

$$
\frac{\partial u_{\tau}}{\partial \tau}=\frac{\partial u_{n}}{\partial \tau}=0
$$

Consequently, by introducing Eqs. (23) and (25) into Eq. (24b), one arrives at

$$
\varepsilon_{\tau \tau}+\mathscr{N}_{1}(w)=0,
$$

where the nonlinear part may be written as

$$
\mathscr{N}_{1}(w)=-\frac{1}{2 h_{\tau}^{2}}\left(\frac{\partial w}{\partial \tau}\right)^{2} .
$$

The above boundary condition can be written in terms of $F$ by combining Eqs. (6) and (5a), yielding

$$
\boldsymbol{\varepsilon}=\frac{1}{h E}[\Delta F \mathbf{1}-(1+v) \boldsymbol{\nabla} \boldsymbol{\nabla} F],
$$


so that

$$
\Delta F-(1+v) \tau \cdot(\nabla \nabla F) \tau+\mathscr{N}_{1}(w)=0 .
$$

The second boundary condition is obtained as a second consequence of Eq. (23), by eliminating all the derivatives with respect to $n$ in the components of $\varepsilon$ so that only derivatives with respect to $\tau$ remain and then vanish, due to the above remark (25). One can verify using Eqs. $(24 a-c)$ that the following expression is valid for any point on the boundary, if Eq. (23) is fulfilled:

$$
\frac{\partial}{\partial n}\left(h_{\tau} \varepsilon_{\tau \tau}\right)-\frac{h_{n} h_{\tau}}{R_{\tau}} \varepsilon_{n n}-2 \frac{\partial}{\partial \tau}\left(h_{n} \varepsilon_{n \tau}\right)+\mathscr{N}_{F}(w)=0
$$

with

$$
\mathscr{N}_{F}(w)=\frac{h_{\tau}}{2 R_{\tau} h_{n}}\left(\frac{\partial w}{\partial n}\right)^{2}+\mathscr{N}_{2}(w)
$$

where $\mathscr{N}_{2}$ is a nonlinear function of $\partial w / \partial \tau$, not written here for the sake of brevity. The above boundary condition (30) can be easily written in terms of $F$ by using Eq. (28). This operation will not be presented here in general curvilinear coordinates, as it leads to very complex expressions. However, the interested reader will find those calculations in case of particular geometries in Appendix C.

A simple practical case is obtained if the transverse displacement $w$ vanishes on the entire boundary $(w=0)$. This assumption is justified as immovable edge in-plane boundary conditions are usually associated with zero transverse displacement, as, for example, in clamped edge, simply supported or hinged edge (see Section 5.2) conditions. As before, it leads to

$$
\frac{\partial w}{\partial \tau}=0
$$

at any point on the edge, so that $\mathscr{N}_{1}$ and $\mathscr{N}_{2}$ vanish in the above two boundary conditions (29) and (30).

Relations (29) and (30) can be considered as two boundary conditions written in terms of $F$. These two equations are necessary to ensure $\mathbf{u}=0$ on $\partial \mathscr{S}$, but not sufficient. To ensure $\mathbf{u}=0$ on $\partial \mathscr{S}$, one would have to integrate in space the expression for $F$ with Eqs. (28) and (3a) to obtain $\mathbf{u}$ that would thus be defined up to an arbitrary rigid body motion. The latter would then be canceled by applying condition Eq. (25). However, Eqs. (29) and (30) written in terms of $F$ are two sufficient boundary conditions if one is interested in transverse displacement $w$ only, as $\mathbf{u}$ appears only through $F$ in the present formulation (Eqs. (36a, b)).

\subsection{Crossed-similarity between the free edge and the clamped edge}

In the case of an edge free of loads in both the transverse and the in-plane direction (classically denoted "free edge" boundary conditions), one has to impose Eqs. (14), (17) and (21). It may be written as

$$
F=0, \quad \frac{\partial F}{\partial n}=0
$$

Eq. (14), Eq. (17).

In the case of an edge clamped in any direction (classically denoted "clamped edge" boundary conditions), one has to impose Eqs. (13), (29) and (30). It appears as

$$
\begin{aligned}
& \text { Eq. (29), Eq. (30), } \\
& w=0, \quad \frac{\partial w}{\partial n}=0 .
\end{aligned}
$$

One can note that Eqs. (33a, b) have the same form as Eqs. $(34 \mathrm{c}, \mathrm{d})$, so that free boundary conditions in the inplane direction written in terms of Airy stress function $F$ have the form of clamped boundary conditions. This result has been previously pointed out in Ref. [40] in the case of a circular plate and is extended here to a plate of arbitrary geometry. 
Conversely, comparing Eqs. (33c,d) and Eqs. (34a,b) is difficult in the general case, because the corresponding expressions in orthogonal curvilinear coordinates are complex. However, this can be done in Cartesian coordinates - c.f. Eqs. (C.3a, b) and (C.4, C.6) - and in polar coordinates - c.f. Eqs. (C.11a, b) and Eqs. (C.12, C.14). Consequently, one can observe that immovable in-plane boundary conditions (in term of $F$ ) and transverse free boundary conditions (in terms of $w$ ) have forms that are very close to each other but not perfectly identical, as some signs differ.

\section{A solution to the nonlinear problem}

\subsection{Dimensionless form of the equations}

Eqs. (7a,b) are rewritten in a dimensionless form by defining the following dimensionless quantities, denoted by overbars:

$$
\begin{gathered}
\mathbf{x}=a \overline{\mathbf{x}}, \quad w=w_{0} \bar{w}, \quad t=a^{2} \sqrt{\rho h / D} \bar{t}, \quad F=E h w_{0}^{2} \bar{F} \\
p=D w_{0} / a^{4} \bar{p}, \quad \mathbf{N}=E h w_{0}^{2} / a^{2} \overline{\mathbf{N}}, \quad \mathbf{M}=D w_{0} / a^{2} \overline{\mathbf{M}} \quad \boldsymbol{\varepsilon}=w_{0}^{2} / a^{2} \overline{\boldsymbol{\varepsilon}}
\end{gathered}
$$

where $a$ is the characteristic length of the mid-surface $\mathscr{S}$ and $w_{0}$ is the order of magnitude of transverse displacement $w$, generally chosen with reference to a particular problem. Substituting the above definitions (Eqs. $(35 \mathrm{a}, \mathrm{b}))$ into the equations of motion $(7 \mathrm{a}, \mathrm{b})$ and dropping the overbars in the result, one obtains

$$
\begin{aligned}
\Delta \Delta w+\ddot{w} & =\varepsilon L(w, F)+p(\mathbf{x}), \\
\Delta \Delta F & =-\frac{1}{2} L(w, w),
\end{aligned}
$$

where $\varepsilon=12\left(1-v^{2}\right) w_{0}^{2} / h^{2}$ and $\ddot{w}$ denotes the second time derivative of $w$. The choices for $w_{0}$ can be $h, h^{2} / a$, $h^{3} / a^{2}$, etc. $w_{0}=h$ is a good choice in the general case, because it enables easy interpretation of the results by comparing the transverse displacement magnitude directly to the thickness of the plate. However, $w_{0}=h^{2} / a$ or $w_{0}=h^{3} / a^{2}$ leads to a small value of $\varepsilon$ compared to unity, if the plate is thin $(h / a \ll 1)$. This latter scaling choice can be useful if perturbation methods are used, as they are justified when the nonlinear terms (scaled by $\varepsilon$ ) are small compared to the linear terms [39,40,43].

\subsection{Mode expansion}

The problem described by Eqs. (7a,b) can be discretized by expanding $w$ and $F$ onto proper expansion functions. A useful set is the eigenmodes of the linear part of Eqs. (7a,b). The main advantage is that the obtained discretized problem has a diagonal linear part.

We denote by $\left(\omega_{k}, \Phi_{k}(\mathbf{x})\right)$ and $\left(\zeta_{k}, \Psi_{k}(\mathbf{x})\right)$ the eigenmodes of the linear part of Eqs. (7a, b) $-\Phi_{k}$ and $\Psi_{k}$ are two functions defined over domain $\mathscr{S}$ - that are solutions of, for all $k \in \mathbb{N}^{*}$ :

$$
\begin{gathered}
\Delta \Delta \Phi_{k}-\omega_{k}^{2} \Phi_{k}=0, \\
\Delta \Delta \Psi_{k}=\zeta_{k}^{4} \Psi_{k}
\end{gathered}
$$

along with proper boundary conditions, chosen in Eqs. (13a, b), (14) and (17) for $\Phi_{k}$ and in Eqs. (21), (29) and (30) for $\Psi_{k}$.

The solution of Eqs. (7a,b) is obtained by using the following expansions for the unknowns $w$ and $F$ :

$$
w(\mathbf{x}, t)=\sum_{k=1}^{+\infty} \Phi_{k}(\mathbf{x}) q_{k}(t), \quad F(\mathbf{x}, t)=\sum_{k=1}^{+\infty} \Psi_{k}(\mathbf{x}) \eta_{k}(t) .
$$




\subsection{Quadratic $(\eta, q)$-formulation}

By introducing Eq. (38a) into Eq. (7b), using Eq. (37b), multiplying the result by $\Psi_{k}$, integrating over the mid-surface $\mathscr{S}$ and finally using the orthogonality properties of modes $\left(\zeta_{k}, \Psi_{k}\right)$, one obtains the membrane modal coordinate $\eta_{k}$ for all $k \in \mathbb{N}^{*}$ :

$$
\eta_{k}(t)=-\frac{1}{2 \zeta_{k}^{4}} \sum_{p=1}^{+\infty} \sum_{q=1}^{+\infty} H_{p q}^{k} q_{p}(t) q_{q}(t)
$$

where

$$
H_{p q}^{k}=\frac{\iint_{\mathscr{S}} \Psi_{k} L\left(\Phi_{p}, \Phi_{q}\right) \mathrm{d} S}{\iint_{\mathscr{S}} \Psi_{k}^{2} \mathrm{~d} S} .
$$

By introducing Eqs. (38a, b) into Eq. (7a), using Eqs. (39) and Eq. (37a), multiplying the result by $\Phi_{k}$, integrating over the mid-surface $\mathscr{S}$ and finally using the orthogonality properties of modes $\left(\omega_{k}, \Phi_{k}\right)$, one obtains the following set of equations, satisfied by the transverse modal coordinates $q_{k}$, for all $k \in \mathbb{N}^{*}$ :

$$
\ddot{q}_{k}(t)+2 \xi_{k} \omega_{k} \dot{q}_{k}(t)+\omega_{k}^{2} q_{k}(t)=\varepsilon \sum_{p=1}^{+\infty} \sum_{q=1}^{+\infty} E_{p q}^{k} q_{p}(t) \eta_{q}(t)+Q_{k}(t)
$$

with

$$
E_{p q}^{k}=\frac{\iint_{\mathscr{S}} \Phi_{k} L\left(\Phi_{p}, \Psi_{q}\right) \mathrm{d} S}{\iint_{\mathscr{S}} \Phi_{k}^{2} \mathrm{~d} S} .
$$

The initial continuous problem of Eqs. $(36 \mathrm{a}, \mathrm{b})$, with proper boundary conditions, has been discretized and now consists in finding modal coordinates $\left(q_{k}, \eta_{k}\right)$, for all $k \in \mathbb{N}^{*}$, solutions of the set of ordinary differential equations (ODEs) (39) and (41). One can remark that the present formulation is quadratic in terms of the $\left\{q_{k}, \eta_{k}\right\}_{k \in \mathbb{N}^{*}}$. A linear viscous modal damping term has also been introduced, with modal damping factor $\xi_{k}$.

\subsection{Cubic q-formulation}

Another formulation can be obtained by eliminating $\eta_{k}$ in Eq. (41) by using its expression as a function of the $\left\{q_{k}\right\}_{k \in \mathbb{N}^{*}}$ (Eq. (39)). Eq. (41) becomes, for all $k \in \mathbb{N}^{*}$ :

$$
\ddot{q}_{k}(t)+2 \xi_{k} \omega_{k} \dot{q}_{k}(t)+\omega_{k}^{2} q_{k}(t)=-\varepsilon \sum_{p=1}^{+\infty} \sum_{q=1}^{+\infty} \sum_{r=1}^{+\infty} \Gamma_{p q r}^{k} q_{p}(t) q_{q}(t) q_{r}(t)+Q_{k}(t)
$$

with

$$
\Gamma_{p q r}^{k}=\sum_{i=1}^{+\infty} \frac{H_{p q}^{i} E_{r i}^{k}}{2 \xi_{i}^{4}} .
$$

In this case, the in-plane unknowns ( $F$, through the in-plane modal coordinates $\left.\left\{\eta_{k}\right\}_{k \in \mathbb{N}^{*}}\right)$ do not appear in the formulation: one has just to calculate the transverse modal coordinates $\left\{q_{k}\right\}_{k \in \mathbb{N}^{*}}$ by solving the set of ODEs (43) and then using the deformed shapes $\left\{\Phi_{k}\right\}_{k \in \mathbb{N}^{*}}$ with Eq. (38a) to obtain $w(\mathbf{x}, t)$. The present formulation has fewer unknowns (only the $\left\{q_{k}\right\}_{k \in \mathbb{N}^{*}}$ ) but, on the other hand, it is cubic in terms of the $\left\{q_{k}\right\}_{k \in \mathbb{N}^{*}}$.

\subsection{Discussion}

A practical advantage of the above choice of the eigenmodes to discretize the nonlinear equations of motions is that the damping can be heuristically introduced by viscous modal damping terms, whose value for each mode must be determined experimentally in any particular application. One obtains a rich damping model, richer than traditional proportional damping approximations, which is essential if one is interested in simulating realistic plate vibration decays [44]. 
In a practical setting, one must truncate the infinite set of Eqs. (38a, b), by retaining $N_{w} \in \mathbb{N}$ functions $\Phi_{k}$ and $N_{F} \in \mathbb{N}$ functions $\Psi_{k}$ in the expansions. This truncation has to be carefully done $[45,46]$ and the number of expansion functions that have to be retained depends on the practical problem under study, in order to obtain a good accuracy of the solution.

The advantage of the second ( $q$ cubic) formulation is that it is more compact, since it introduces fewer unknowns (only $N_{w}$ unknowns $q_{k}$ in Eq. (43)). It has been successfully used in many past studies of nonlinear vibrations of plates and shells. The interested reader is referred to the following books for thorough literature reviews $[2,16,28]$. The cubic- $q$ formulation has also served as a basis for numerical simulations in more recent studies. Among others, the interested reader can refer to the above-cited textbook [28] and to [46,47], that present various numerical studies that solve similar cubic sets of ODEs. Direct time integrations as well as amplitude/frequency diagrams obtained with the continuation software AUTO [48] are presented.

On the contrary, the first $(\eta, q)$ formulation has the advantage of being quadratic in terms of the unknowns. Even if more unknowns have to be considered ( $N_{F}$ unknowns $\eta_{k}$ in Eq. (39) plus $N_{w}$ unknowns $q_{k}$ in Eq. (41)), some resolution methods mandatory need a quadratic formulation of the problem. This is the case of the Asymptotic Numerical Method [49,50], a powerful continuation method that has recently been implemented in the Matlab software environment, under the name MANLab [51,52]. Other interesting examples are some energy-conserving schemes, developed by the second author [36] in the time-space (i.e. non-modal) context, but applicable to systems of ODEs as well. In both formulations, the number of time integrations is the same: $N_{w}$ second-order nonlinear ODEs have to be integrated, since Eq. (39) is an algebraic nonlinear set of equations.

Another remark is that any eigenmode $\Psi_{k}$ solution of Eq. (37b) associated with two boundary conditions chosen in Eqs. (21), (29) and (30) is uniquely determined. As a consequence, $F$, calculated with relation (38b), is also perfectly defined, with $\eta_{k}$ and $q_{k}$ solutions of Eqs. (39) and (41). $F$ obtained in this way then constitutes one solution of the set $(36 \mathrm{a}, \mathrm{b})$, which is not the only one, since an $F$ solution of $(36 \mathrm{a}, \mathrm{b})$ is defined up to an arbitrary bilinear space function (see Section 2.2).

\section{Triple self-adjointness of the von Kármán operator}

In this section, a particular property of $L(\circ, \circ)$ is investigated in the general case of a plate boundary of arbitrary geometry. This property has been introduced and called "triple self-adjointness" in Ref. [36] in the case of a rectangular plate. It states that for scalar field $f, g$ and $h$, from $\mathscr{S}$ into $\mathbb{R}$, one has

$$
\iint_{\mathscr{S}} f L(g, h) \mathrm{d} S=\iint_{\mathscr{S}} L(f, g) h \mathrm{~d} S+\oint_{\partial \mathscr{S}} I \mathrm{~d} s,
$$

where $I$ is a function of $f, g$ and $h$ and their spatial derivatives.

The purpose of this section is to prove the above property and to find the expression for $I$, in the case of a boundary of arbitrary shape. Moreover, it will be shown that $I$ vanishes for some particular boundary conditions.

\subsection{General case}

One can first remark that, by using identities (B.14) twice and (B.17):

$$
\begin{aligned}
f \Delta h & =f \operatorname{div}(\boldsymbol{\nabla} h) \\
& =\operatorname{div}(f \nabla h)-\boldsymbol{\nabla} f \cdot \boldsymbol{\nabla} h \\
& =\operatorname{div}(f \nabla h)-\operatorname{div}(h \boldsymbol{\nabla} f)+h \operatorname{div}(\nabla f),
\end{aligned}
$$

so that

$$
f \Delta h=h \Delta f+\operatorname{div}(f \nabla h-h \boldsymbol{\nabla} f) .
$$


Then, using the same method and identities (B.14), (B.17) and (B.21), one has, for any vector field v:

$$
\Delta g \operatorname{div} \mathbf{v}=\operatorname{div}(\Delta g \mathbf{v})-\mathbf{v} \operatorname{div}(\nabla \nabla g) .
$$

Finally, by multiplying Eq. (49) by $\Delta g$ and using Eq. (50) by substituting $f \nabla h-h \nabla f$ for $\mathbf{v}$, one obtains

$$
f \Delta g \Delta h=h \Delta f \Delta g+\operatorname{div}[\Delta g(f \nabla h-h \nabla f)]-(f \nabla h-h \nabla f) \cdot \operatorname{div}(\nabla \nabla g) .
$$

In the same manner as above, by using identity (B.19) twice, one can show that

$$
f \nabla \nabla h=h \nabla \nabla f+\nabla(f \nabla h-h \nabla f) .
$$

Finally, by multiplying Eq. (52) by $\nabla \nabla g$ and using identity Eq. (B.18) by substituting $\nabla \nabla g$ for $\mathbf{T}$ and $f \nabla h-$ $h \boldsymbol{\nabla} f$ for $\mathbf{u}$, one obtains

$$
f \nabla \nabla g: \nabla \nabla h=h \nabla \nabla g: \nabla \nabla f+\operatorname{div}[\nabla \nabla g(f \nabla h-h \nabla f)]-\operatorname{div}(\nabla \nabla g) \cdot(f \nabla h-h \nabla f) .
$$

Now, by considering the definition (Eq. (8)) of the von Kármán operator $L(\circ, \circ)$ and using Eqs. (51) and (53), one obtains the following compact formula:

$$
f L(g, h)=L(f, g) h+\operatorname{div}[(\Delta g \mathbf{1}-\boldsymbol{\nabla} \boldsymbol{\nabla} g)(f \boldsymbol{\nabla} h-h \boldsymbol{\nabla} f)] .
$$

Then, by integrating the above formula over domain $\mathscr{S}$ and applying the divergence theorem, one obtains the triple self-adjointness property (45), with

$$
I=[(\Delta g \mathbf{1}-\nabla \nabla g)(f \nabla h-h \nabla f)] \cdot \mathbf{n} .
$$

\subsection{A particular simplification}

The purpose of this section is to investigate which of the classical boundary conditions leads to the vanishing of $I(\mathbf{x})$ for any $\mathbf{x} \in \partial \mathscr{S}$. In this case, one has

$$
\iint_{\mathscr{S}} f L(g, h) \mathrm{d} S=\iint_{\mathscr{S}} L(f, g) h \mathrm{~d} S
$$

that simplifies the calculation of the coefficients that appear in the modal expansion introduced in Section 4, as some of them become equal. In fact, if Eq. (56) holds, it is obvious with Eqs. (40), (42) and (44) that for any integer $p, q, r, k$ :

$$
E_{p k}^{q}=H_{p q}^{k}, \quad \Gamma_{p q r}^{k}=\sum_{i=1}^{+\infty} \frac{H_{p q}^{i} H_{r k}^{i}}{2 \xi_{i}^{4}}
$$

provided the mode shapes have been normalized in the following way, for all $k$ :

$$
\iint_{\mathscr{S}} \Phi_{k}^{2} \mathrm{~d} S=\iint_{\mathscr{S}} \Psi_{k}^{2} \mathrm{~d} S=1 .
$$

The above simplification can be very useful because all coefficients $E_{p k}^{q}$ and $H_{p q}^{k}$ are numerically evaluated in a practical case. If $N_{F} \times N_{w}$ functions $\Psi_{k} \times \Phi_{k}$ are retained, considering the symmetry of the von Kármán operator $(L(f, g)=L(g, f))$, one has to numerically evaluate $N=N_{F} N_{w}\left(N_{w}+1\right) / 2$ coefficients, a number that can be large in a practical case (If $N_{F}=N_{w}=30, N=13950$ ). If Eq. (57) holds, only half of the coefficients have to be calculated, which saves half the computation time.

As a preliminary step, $I(\mathbf{x})$ is written in the orthogonal curvilinear coordinate system defined in Section 3.2, with Eqs. (B.9), (B.15) and (B.16) of the appendix. One obtains

$$
\begin{aligned}
I= & \frac{1}{h_{n}}\left[\frac{1}{h_{t}^{2}} \frac{\partial^{2} g}{\partial \tau^{2}}+\frac{1}{h_{n} R_{\tau}} \frac{\partial g}{\partial n}-\frac{1}{h_{\tau}^{3}} \frac{\partial h_{\tau}}{\partial \tau} \frac{\partial g}{\partial \tau}\right]\left(f \frac{\partial h}{\partial n}-h \frac{\partial f}{\partial n}\right) \\
& -\frac{1}{h_{\tau}}\left[\frac{1}{h_{n} h_{\tau}} \frac{\partial^{2} g}{\partial n \partial \tau}-\frac{1}{h_{n} R_{n}} \frac{\partial g}{\partial n}-\frac{1}{h_{\tau} R_{\tau}} \frac{\partial g}{\partial \tau}\right]\left(f \frac{\partial h}{\partial \tau}-h \frac{\partial f}{\partial \tau}\right)
\end{aligned}
$$


Then, two simple cases lead to a vanishing of $I$ at any point on the boundary:

$$
\text { either } f=\frac{\partial f}{\partial n}=\frac{\partial f}{\partial \tau}=0 \quad \text { or } \quad h=\frac{\partial h}{\partial n}=\frac{\partial h}{\partial \tau}=0
$$

at any point on the boundary. As we are interested in proving Eqs. (57), considering Eq. (40), $f$ plays the role of Airy stress function $F$ (or any in-plane mode $\Psi_{k}$ ); $g$, and $h$ play the role of transverse displacement $w$ (or any transverse mode $\Phi_{k}$ ). Thus, Eq. (57) is fulfilled in the following two cases:

- if the edge free of loads in the in-plane direction, since this case is obtained if

$$
F=\frac{\partial F}{\partial n}=0
$$

at any point on the edge (see Section 3.2.1);

- if the edge is clamped in the transverse direction, since this case is obtained if

$$
w=\frac{\partial w}{\partial n}=0
$$

at any point on the edge.

As in Section 3.2.1, we have considered that $f=\partial f / \partial n=0$ at any point at the edge necessarily implies that $\partial f / \partial \tau=0$, for any scalar field $f$. The above two cases obviously show that classical clamped-edge as well as free-edge boundary conditions necessarily implied property Eq. (57).

The particular case of hinged-edge boundary conditions has to be addressed. As the nonlinearities couple transverse- $w$ and in-plane- $F$ motion, one has to consider two subcases.

- The case of a simply supported edge is obtained if

$$
w=M_{n n}=0
$$

and if the edge is free of loads in the plane (Eqs. $(61 \mathrm{a}, \mathrm{b}))$. In this case, because of this latter reason, property Eq. (57) is fulfilled.

- The case of a hinged edge is obtained if Eqs. $(63 a, b)$ are fulfilled and if the edge is immovable in the plane (Eqs. (29) and (30)). Considering the corresponding expressions as a function of $w$ and $F$, it appears that

Table 1

Summary of the classical homogeneous boundary conditions and the associated relations in terms of $w$ and $F$

$\begin{array}{lll}\text { Rot. immov. } & \begin{array}{l}\text { Rot. free } \\ \text { Eq. (17) }\end{array} & \text { Transversely free Eq. (14) } \\ \text { In-plane immovable } \\ \begin{array}{l}\text { Eqs. (29) and (30) } \\ F=\partial F / \partial n=0\end{array}\end{array}$

The cases where the simplified TSA property (56) holds are denoted by "STSA". 
they cannot generally lead to the vanishing of $I$. As a consequence, in the case of a hinged edge, property Eq. (57) is generally not fulfilled.

As a consequence, three classical cases lead to the vanishing of $I$ : a free edge, a clamped edge and a simply supported edge. The case of a clamped edge in the transverse direction with a free edge in the in-plane direction, that also leads to the vanishing of $I$, has already been shown in Ref. [53]. The case of a hinged edge does not, in general, simplify Eq. (45) to Eq. (56). These results are gathered in Table 1.

\section{Conclusion}

The main goal of the paper has been to exhibit some properties of the von Kármán model of nonlinear mechanical behavior of thin plates. As a preliminary, the main steps of the derivation of the two governing partial differential equations have been written in terms of two unknown scalar fields: the transverse displacement $w$ and an Airy stress function $F$. To obtain a general and compact formulation, intrinsic notations have been used, so that all the important relations can be written in any particular coordinate system, depending on the plate edge shape. This can be easily done by using the formula giving the classical differential operators in a particular coordinate system, to be found in many textbooks. As an example, an appendix gathers all important formulae, written in Cartesian and polar coordinates.

As the nonlinearities couple the transverse and in-plane motions, one has to consider in-plane boundary conditions (BC) even if only the transverse behavior of the plate is addressed. As a consequence, in-plane BC in terms of $F$ have been derived for a plate edge of arbitrary geometry, in the particular cases of a free edge and an immovable edge. In the case of a free edge, it has been shown that the in-plane $\mathrm{BC}$ in terms of $F$ take the form of clamped boundary conditions. In the case of an immovable edge, the in-plane boundary conditions show complicated expressions in terms of $F$, close, but not identical, to free BC. An intrinsic expression, written in terms of the membrane strains for the sake of brevity and that can be easily rewritten in terms of $F$, has been given.

Then, the governing partial differential equations have been discretized by expanding the two unknown scalar fields $w$ and $F$ onto the eigenmodes of the associated linear problem. Two discretized formulations have been exhibited, in terms of two sets of nonlinear ordinary differential equations (ODE). The first one is quadratic in terms of the unknowns, an essential property if those ODE have to be integrated by the asymptotic numerical method, such as a continuation method [49,50]. This quadratic form is also useful in the context of some energy-conserving numerical schemes [36]. A second formulation, cubic in terms of the unknowns and already used in past studies on plates, has also been obtained.

Finally, a particular property of the so-called von Kármán bilinear operator has been given and proved, again for a plate edge of arbitrary geometry, thanks to the intrinsic formulation. It is shown that this property, called the triple self-adjointness, can lead to simplifications that enable to divide by a factor two the computation time needed for the numerical coefficients of the ODE set calculation. This property has also been found to be essential to use the above-cited energy-conserving numerical schemes [36].

\section{Acknowledgments}

The long-time research companion and friend of the first author, Cyril Touzé, is heartily thanked for his long-time support and friendship and especially for organizing the meeting of the authors. Roger Ohayon is also warmly thanked for fruitful discussions about the Airy stress function.

\section{Appendix A. Notations for operators}

In this article, we use the same notation $\mathscr{P}$ for the plate mid-plane - a two-dimensional Euclidean spaceand the associated two-dimensional vector space.

$\mathbf{u} \cdot \mathbf{v}$ denotes the scalar (dot) product between two vectors $\mathbf{u}$ and $\mathbf{v}$. In an orthonormal coordinate system, it writes $\mathbf{u} \cdot \mathbf{v}=u_{\alpha} v_{\alpha}$. 
$\mathbf{u} \otimes \mathbf{v}$ denotes the tensor (dyadic) product between vectors $\mathbf{u}$ and $\mathbf{v}$. If $\mathbf{T}=\mathbf{u} \otimes \mathbf{v}$, where $\mathbf{u}, \mathbf{v} \in \mathscr{P}$, one defines the linear transformation $\mathbf{w} \in \mathscr{P} \mapsto \mathbf{T w}=(\mathbf{v} \cdot \mathbf{w}) \mathbf{u} \in \mathscr{P}$. The components of tensor $\mathbf{T}$ on the basis $\left(\mathbf{e}_{1}, \mathbf{e}_{2}\right)$ of $\mathscr{P}$ are $T_{\alpha \beta}=u_{\alpha} v_{\beta}$. The obtained tensor is a second-order tensor, an element of a four-order vector space written $\mathscr{P} \otimes \mathscr{P}$. One usually writes $\mathbf{T}=T_{\alpha \beta} e_{\alpha} \otimes e_{\beta}$.

$\mathbf{S}: \mathbf{T}$ denotes the doubly contracted (double dot) product of tensors $\mathbf{S}$ and $\mathbf{T}$, that writes in an orthonormal coordinate system $\mathbf{S}: \mathbf{T}=S_{\alpha \beta} T_{\beta \alpha}$.

$\boldsymbol{\nabla} a$ and $\boldsymbol{\nabla u}$ denote, respectively, the vector gradient of scalar field $a$ and the tensor gradient of vector field $\mathbf{u}$. $\operatorname{div} \mathbf{u}$ and $\operatorname{div} \mathbf{T}$ denote, respectively, the scalar divergence of vector field $\mathbf{u}$ and the vector divergence of tensor field T. $\Delta a$ is the Laplacian of scalar field $a$ and $\nabla \nabla a$ is the tensor gradient of the vector gradient of scalar field $a$, also called the Hessian. All these differential operators are defined in Appendix B.

\section{Appendix B. Differential operators in orthogonal curvilinear coordinates}

The aim of this section is to recall the main properties of orthogonal curvilinear coordinates in the plane in order to derive the expressions of the main differential operators (Fig. B.1).

\section{B.1. Curvilinear orthogonal coordinates}

The position vector $\mathbf{x}$ of any point $M$ of a given region of plane $\mathscr{P}$ is defined by two curvilinear coordinates $\left(v_{1}, v_{2}\right) \in I_{1} \times I_{2}$, with $I_{1} \times I_{2} \subset \mathbb{R}^{2}$, so that $\mathbf{x}=\mathbf{O M}=\mathbf{f}\left(v_{1}, v_{2}\right)$, where $\mathbf{f}:\left(v_{1}, v_{2}\right) \in I_{1} \times I_{2} \mapsto \mathbf{f}\left(v_{1}, v_{2}\right) \in \mathscr{P}$ is a mapping from $I_{1} \times I_{2}$ into $\mathscr{P}$ that defines the curvilinear coordinate system.

Let two basis vectors of plane $\mathscr{P}$ be defined by

$$
\mathbf{g}_{\alpha}=\frac{\partial \mathbf{f}}{\partial v_{\alpha}}, \quad \alpha \in\{1,2\}
$$

The two families of coordinate lines are defined by

$$
\begin{aligned}
& \mathscr{C}_{1}=\mathscr{C}_{1}\left(v_{20}\right)=\left\{M \in \mathscr{P} / \mathbf{O M}=\mathbf{f}\left(v, v_{20}\right), v \in I_{1}\right\}, \\
& \mathscr{C}_{2}=\mathscr{C}_{2}\left(v_{10}\right)=\left\{M \in \mathscr{P} / \mathbf{O M}=\mathbf{f}\left(v_{10}, v\right), v \in I_{2}\right\} .
\end{aligned}
$$

One can note that $\mathbf{g}_{\alpha}, \alpha \in\{1,2\}$, is tangential to $\mathscr{C}_{\alpha}$.

We now assume that $\mathscr{C}_{1}$ and $\mathscr{C}_{2}$ form a family of orthogonal curves, so that for all $\left(\alpha_{1}, \alpha_{2}\right) \in I_{1} \times I_{2}, \mathbf{g}_{1}$ is normal to $\mathbf{g}_{2}$. It is convenient to use a basis of unit vectors, defined by

$$
\mathbf{e}_{\alpha}=\frac{\mathbf{g}_{\alpha}}{\left\|\mathbf{g}_{\alpha}\right\|}=\frac{1}{h_{\alpha}} \frac{\partial \mathbf{f}}{\partial v_{\alpha}}, \quad \alpha \in\{1,2\}
$$

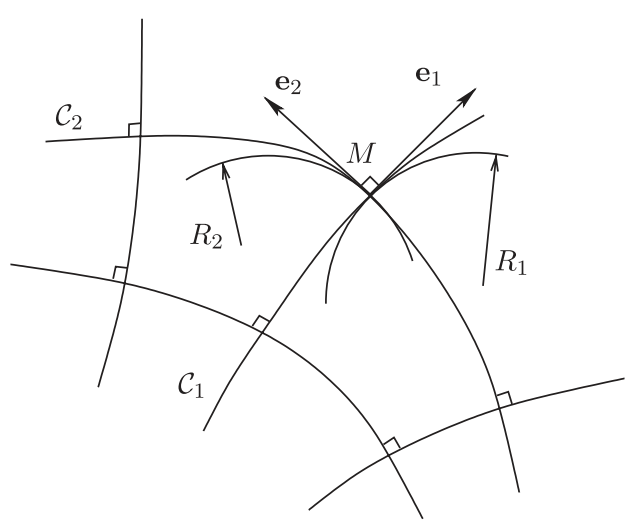

Fig. B.1. Sketch of the curvilinear coordinate system. 
where

$$
h_{\alpha}=h_{\alpha}\left(v_{1}, v_{2}\right)=\left\|\frac{\partial \mathbf{f}}{\partial v_{\alpha}}\right\|, \quad \alpha \in\{1,2\}
$$

are scale factors. By applying the classical Frenet relations between the radius of curvature and the tangent and normal unit vectors of a plane curve [54] to orthogonal curves $\mathscr{C}_{1}$ and $\mathscr{C}_{2}$, one obtains the following properties:

$$
\begin{aligned}
& \frac{\partial \mathbf{e}_{1}}{\partial v_{1}}=\frac{h_{1}}{R_{1}} \mathbf{e}_{2}, \quad \frac{\partial \mathbf{e}_{2}}{\partial v_{2}}=-\frac{h_{2}}{R_{2}} \mathbf{e}_{1}, \\
& \frac{\partial \mathbf{e}_{2}}{\partial v_{1}}=-\frac{h_{1}}{R_{1}} \mathbf{e}_{1}, \quad \frac{\partial \mathbf{e}_{1}}{\partial v_{2}}=\frac{h 2}{R_{2}} \mathbf{e}_{2},
\end{aligned}
$$

where $R_{\alpha}=R_{\alpha}\left(v_{1}, v_{2}\right), \alpha \in\{1,2\}$, is the radius of curvature of curve $\mathscr{C}_{\alpha}$. The signs in Eqs. (B.6a, b) have been chosen so that $R_{1}>0\left(R_{2}<0\right)$ if $\mathbf{x}_{2}\left(\mathbf{x}_{1}\right)$ points toward the center of curvature of $\mathscr{C}_{1}\left(\mathscr{C}_{2}\right)$. One can also show that

$$
\frac{\partial h_{2}}{\partial v_{1}}=\frac{h_{1} h_{2}}{R_{2}}, \quad \frac{\partial h_{1}}{\partial v_{2}}=-\frac{h_{1} h_{2}}{R_{1}}
$$

\section{B.2. Differential operators}

In the following, $\mathbf{x}=\mathbf{f}\left(v_{1}, v_{2}\right)$ denotes the position vector of any point $M$ of plane $\mathscr{P},\left(v_{1}, v_{2}\right)$ its curvilinear coordinates and $\left(\mathbf{e}_{1}, \mathbf{e}_{2}\right)$ the orthonormal curvilinear basis defined in Section B.1. $a$ denotes a two-dimensional scalar field, from $\mathscr{P}$ into $\mathbb{R}, \mathbf{u}$ denotes a two-dimensional vector field from $\mathscr{P}$ into $\mathscr{P}$, defined by $\mathbf{u}=u_{\alpha} \mathbf{e}_{\alpha}$ and $\mathbf{T}$ denotes a two-dimensional tensor field, from $\mathscr{P}$ into $\mathscr{P} \otimes \mathscr{P}$, defined by $\mathbf{T}=T_{\alpha \beta} \mathbf{e}_{\alpha} \otimes \mathbf{e}_{\beta}$.

\section{B.2.1. Vector gradient}

By definition, the gradient of $a$ is a vector field of $\mathscr{P}$, denoted by $\nabla a$, such that

$$
\mathrm{d} a=\nabla a \cdot \mathrm{d} \mathbf{x} .
$$

To calculate the components of $\boldsymbol{\nabla} a$ in basis $\left(\mathbf{e}_{1}, \mathbf{e}_{2}\right)$, one can differentiate $a$ and $\mathbf{x}$, introduce the result into Eq. (B.8) and identify the two members of the obtained equation, to obtain

$$
\nabla a=\frac{1}{h_{\alpha}} \frac{\partial a}{\partial v_{\alpha}} \mathbf{e}_{\alpha}
$$

\section{B.2.2. Tensor gradient}

By definition, the gradient of $\mathbf{u}$ is a tensor field of $\mathscr{P} \otimes \mathscr{P}$, denoted by $\boldsymbol{\nabla} \mathbf{u}$, such that

$$
\mathrm{d} \mathbf{u}=\boldsymbol{\nabla} \mathbf{u} \mathrm{d} \mathbf{x} .
$$

In a similar way as in the previous section, by differentiating $\mathbf{u}$, one obtains the components of the tensor gradient in basis $\left(\mathbf{e}_{1}, \mathbf{e}_{2}\right)$ :

$$
\boldsymbol{\nabla u}:\left(\begin{array}{cc}
\frac{1}{h_{1}} \frac{\partial u_{1}}{\partial v_{1}}-\frac{u_{2}}{R_{1}} & \frac{1}{h_{2}} \frac{\partial u_{1}}{\partial v_{2}}-\frac{u_{2}}{R_{2}} \\
\frac{1}{h_{1}} \frac{\partial u_{2}}{\partial v_{1}}+\frac{u_{1}}{R_{1}} & \frac{1}{h_{2}} \frac{\partial u_{2}}{\partial v_{2}}+\frac{u_{1}}{R_{2}}
\end{array}\right)_{\left(\mathbf{e}_{1}, \mathbf{e}_{2}\right)}
$$




\section{B.2.3. Scalar divergence and Laplacian}

The scalar divergence of vector field $\mathbf{u}$ can be defined by

$$
\operatorname{div} \mathbf{u}=\operatorname{tr}(\nabla \mathbf{u}) .
$$

With Eq. (B.11), one obtains

$$
\operatorname{div} \mathbf{u}=\frac{1}{h_{1}} \frac{\partial u_{1}}{\partial v_{1}}-\frac{u_{2}}{R_{1}}+\frac{1}{h_{2}} \frac{\partial u_{2}}{\partial v_{2}}+\frac{u_{1}}{R_{2}} .
$$

The Laplacian of scalar field $a$ is, by definition:

$$
\Delta a=\operatorname{div}(\nabla a) .
$$

With Eqs. (B.9) and (B.13), one obtains

$$
\left.\left.\Delta a=\frac{1}{h_{1}^{2}} \frac{\partial^{2} a}{\partial v_{1}^{2}}+\frac{1}{h_{2}^{2}} \frac{\partial^{2} a}{\partial v_{2}^{2}}+\frac{1}{h_{1}} \frac{1}{R_{2}}-\frac{1}{h_{1}^{2}} \frac{\partial h_{1}}{\partial v_{1}}\right) \frac{\partial a}{\partial v_{1}}-\frac{1}{h_{2}} \quad \frac{1}{R_{1}}+\frac{1}{h_{2}^{2}} \frac{\partial h_{2}}{\partial v_{2}}\right) \frac{\partial a}{\partial v_{2}} .
$$

\section{B.2.4. Another useful operator (the Hessian)}

Using Eqs. (B.9), (B.11) and properties (B.7), one can show that

$$
\begin{aligned}
\boldsymbol{\nabla} \nabla a= & \left.\frac{1}{h_{1}^{2}} \frac{\partial^{2} a}{\partial v_{1}^{2}}-\frac{1}{h_{1}^{3}} \frac{\partial h_{1}}{\partial v_{1}} \frac{\partial a}{\partial v_{1}}-\frac{1}{R_{1} h_{2}} \frac{\partial a}{\partial v_{2}}\right) \mathbf{e}_{1} \otimes \mathbf{e}_{1} \\
& \left.+\frac{1}{h_{2}^{2}} \frac{\partial^{2} a}{\partial v_{2}^{2}}-\frac{1}{h_{2}^{3}} \frac{\partial h_{2}}{\partial v_{2}} \frac{\partial a}{\partial v_{2}}+\frac{1}{R_{2} h_{1}} \frac{\partial a}{\partial v_{1}}\right) \mathbf{e}_{2} \otimes \mathbf{e}_{2} \\
& +\left(\frac{1}{h_{1} h_{2}} \frac{\partial^{2} a}{\partial v_{1} v_{2}}-\frac{1}{R_{1} h_{1}} \frac{\partial a}{\partial v_{1}}-\frac{1}{R_{2} h_{2}} \frac{\partial a}{\partial v_{2}}\right)\left(\mathbf{e}_{1} \otimes \mathbf{e}_{2}+\mathbf{e}_{2} \otimes \mathbf{e}_{1}\right)
\end{aligned}
$$

\section{B.2.5. Useful identities}

Here are some classical identities:

$$
\begin{gathered}
\operatorname{div}(a \mathbf{u})=a \operatorname{div} \mathbf{u}+\mathbf{u} \cdot \boldsymbol{\nabla} a, \\
\operatorname{div}(\mathbf{T u})=\mathbf{T}: \boldsymbol{\nabla} \mathbf{u}+\operatorname{div} \mathbf{T}^{\mathrm{T}} \cdot \mathbf{u}, \\
\boldsymbol{\nabla}(a \mathbf{u})=a \boldsymbol{\nabla} \mathbf{u}+\mathbf{u} \otimes \nabla a, \\
\boldsymbol{\nabla} \operatorname{div} \mathbf{u}=\operatorname{div} \nabla^{\mathrm{T}} \mathbf{u}, \\
\boldsymbol{\nabla}^{\mathrm{T}} \boldsymbol{\nabla} a=\boldsymbol{\nabla} \boldsymbol{\nabla} a, \\
\operatorname{div}(a \mathbf{1})=\boldsymbol{\nabla} a .
\end{gathered}
$$

\section{Appendix C. Application to particular geometries}

In this section, the main formulae, written in an intrinsic form in the core of the paper, are translated into a special coordinate system, to be applied to, respectively, a rectangular and a circular plate.

\section{C.1. A rectangular plate}

A rectangular plate is considered. A rectangular Cartesian $(x, y)$ orthonormal coordinate system is used (see Fig. C.1), defined by the mapping

$$
\mathbf{O M}=\mathbf{f}(x, y)=x \mathbf{e}_{x}+y \mathbf{e}_{y} .
$$



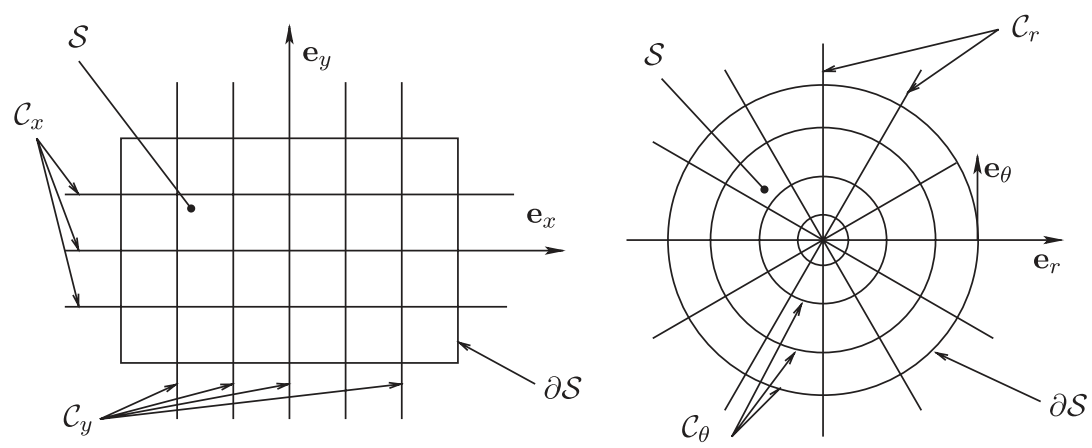

Fig. C.1. Sketch of the two particular geometries with the associated coordinate system.

The coordinate lines are straight (with zero curvatures: $1 / R_{x}=1 / R_{y}=0$ ) and $h_{x}=\|\partial \mathbf{f} / \partial x\|=$ $h_{y}=\|\partial \mathbf{f} / \partial y\|=1$.

The edge of the plate is thus composed of particular coordinate lines, parallel to $\mathbf{e}_{x}$ and $\mathbf{e}_{y}$ (Fig. C.1).

\section{C.1.1. Classical boundary conditions}

The classical boundary conditions in terms of $w$ and $F$ can be any combination of the following relations that must be satisfied at any point on the edge $\partial \mathscr{S}$ :

- In-plane direction:

$\circ$ Free edge (Eqs. $(21 \mathrm{a}, \mathrm{b}))$ :

$$
F=\frac{\partial F}{\partial n}=0
$$

○ Immovable edge, with $w=0$ on the boundary, so that $\mathscr{N}_{1}=\mathscr{N}_{2}=0$ (Eqs. (29) and (30)):

$$
\frac{\partial^{2} F}{\partial n^{2}}-v \frac{\partial^{2} F}{\partial \tau^{2}}=0, \quad \frac{\partial^{3} F}{\partial n^{3}}+(2+v) \frac{\partial^{3} F}{\partial n \partial \tau^{2}}=0 .
$$

- Edge rotation:

$\circ$ Rotationally free edge (Eq. (17)):

$$
\frac{\partial^{2} w}{\partial n^{2}}+v \frac{\partial^{2} w}{\partial \tau^{2}}=0
$$

○ Rotationally immovable edge:

$$
\frac{\partial w}{\partial n}=0
$$

- Transverse edge translation:

$\circ$ Free edge (Eq. (14)):

$$
\frac{\partial^{3} w}{\partial n^{3}}+(2-v) \frac{\partial^{3} w}{\partial n \partial \tau^{2}}+\mathscr{N}_{w}(F, w)=0
$$

with

$$
\mathscr{N}_{w}(F, w)=-\frac{1}{D}\left(\frac{\partial^{2} F}{\partial \tau^{2}} \frac{\partial w}{\partial n}-\frac{\partial^{2} F}{\partial n \partial \tau} \frac{\partial w}{\partial \tau}\right) .
$$


○ Immovable edge:

$$
w=0 .
$$

In all the above relations, $\tau=x(\tau=y)$ and $n=y(n=x)$ if the considered edge is parallel to $\mathbf{e}_{x}\left(\mathbf{e}_{y}\right)$.

\section{C.1.2. Triple self-adjointness property}

It is written as

$$
\begin{aligned}
\iint_{\mathscr{S}} f L(g, h) \mathrm{d} S= & \iint_{\mathscr{S}} L(f, g) h \mathrm{~d} S \\
& +\oint_{\partial \mathscr{S}}\left[\frac{\partial^{2} g}{\partial \tau^{2}}\left(f \frac{\partial h}{\partial n}-h \frac{\partial f}{\partial n}\right)-\frac{\partial^{2} g}{\partial \tau \partial n}\left(f \frac{\partial h}{\partial \tau}-h \frac{\partial f}{\partial \tau}\right)\right] \mathrm{d} \tau
\end{aligned}
$$

with the same rule on $n$ and $\tau$ corresponding to $x$ and $y$ as before.

\section{C.2. A circular plate}

A circular plate of radius $a$ is considered. A polar orthonormal coordinate system $(r, \theta)$ is used (see Fig. C.1, defined by the mapping

$$
\mathbf{O M}=\mathbf{f}(r, \theta)=r \mathbf{e}_{r} .
$$

$C_{r}$ coordinate lines are straight (with zero curvatures: $1 / R_{r}=0$ ) and $C_{\theta}$ coordinate lines are concentric circles, of radius $r . h_{r}=\|\mathrm{\partial} \mathbf{f} / \partial r\|=1$ and $h_{\theta}=\|\partial \mathbf{f} / \partial \theta\|=r$.

The edge of the plate is the coordinate line $\mathscr{C}_{\theta}$ of radius $a$, of tangent and normal vectors $\tau=\mathbf{e}_{\theta}$ and $\mathbf{n}=\mathbf{e}_{r}$ (Fig. C.1).

\section{C.2.1. Classical boundary conditions}

The classical boundary conditions in terms of $w$ and $F$ can be any combination of the following relations that must be satisfied at any point on the edge $\partial \mathscr{S}$ :

- In-plane direction:

$\circ$ Free edge Eqs. (21):

$$
F=\frac{\partial F}{\partial r}=0
$$

○ Immovable edge, with $w=0$ on the boundary, so that $\mathscr{N}_{1}=\mathscr{N}_{2}=0$ (Eqs. (29) and (30)):

$$
\begin{gathered}
\frac{\partial^{2} F}{\partial r^{2}}-v\left(\frac{1}{r} \frac{\partial F}{\partial r}+\frac{1}{r^{2}} \frac{\partial^{2} F}{\partial \theta^{2}}\right)=0 \\
\frac{\partial^{3} F}{\partial r^{3}}+\frac{1}{r} \frac{\partial^{2} F}{\partial r^{2}}-\frac{1}{r^{2}} \frac{\partial F}{\partial r}+\frac{2+v}{r^{2}} \frac{\partial^{3} F}{\partial r \partial \theta^{2}}-\frac{3+v}{r^{3}} \frac{\partial^{2} F}{\partial \theta^{2}}+\mathscr{N}_{F}(w)=0
\end{gathered}
$$

with

$$
\mathscr{N}_{F}(w)=\frac{1}{2 r}\left(\frac{\partial^{2} w}{\partial r^{2}}\right)^{2}
$$

- Edge rotation:

○ Rotationally free edge (Eq. (17)):

$$
\frac{\partial^{2} w}{\partial r^{2}}+v\left(\frac{1}{r} \frac{\partial w}{\partial r}+\frac{1}{r^{2}} \frac{\partial^{2} w}{\partial \theta^{2}}\right)=0 .
$$


- Rotationally immovable edge:

$$
\frac{\partial w}{\partial r}=0
$$

- Transverse edge translation:

$\circ$ Free edge (Eq. (14)):

$$
\frac{\partial^{3} w}{\partial r^{3}}+\frac{1}{r} \frac{\partial^{2} w}{\partial r^{2}}-\frac{1}{r^{2}} \frac{\partial w}{\partial r}+\frac{2-v}{r^{2}} \frac{\partial^{3} w}{\partial r \partial \theta^{2}}-\frac{3-v}{r^{3}} \frac{\partial^{2} w}{\partial \theta^{2}}+\mathscr{N}_{w}(F, w)=0
$$

with

$$
\mathscr{N}_{w}(F, w)=-\frac{1}{D}\left(\frac{1}{r} \frac{\partial F}{\partial r}+\frac{1}{r^{2}} \frac{\partial^{2} F}{\partial \theta^{2}}\right) \frac{\partial w}{\partial r}+\frac{1}{D}\left(\frac{1}{r} \frac{\partial F}{\partial r \partial \theta}-\frac{1}{r^{2}} \frac{\partial F}{\partial \theta}\right) \frac{1}{r} \frac{\partial w}{\partial \theta}
$$

- Immovable edge:

$$
w=0 .
$$

\section{C.2.2. Triple self-adjointness property}

It may be written as

$$
\begin{aligned}
\iint_{\mathscr{S}} f L(g, h) \mathrm{d} S= & \iint_{\mathscr{S}} L(f, g) h \mathrm{~d} S+\int_{0}^{2 \pi}\left[\left(\frac{\partial g}{\partial r}+\frac{1}{r} \frac{\partial^{2} g}{\partial \theta^{2}}\right)\left(f \frac{\partial h}{\partial r}-h \frac{\partial f}{\partial r}\right)\right. \\
& \left.-\frac{1}{r}\left(\frac{\partial^{2} g}{\partial r \partial \theta}-\frac{1}{r} \frac{\partial g}{\partial \theta}\right)\left(f \frac{\partial h}{\partial \theta}-h \frac{\partial f}{\partial \theta}\right)\right] \mathrm{d} \theta .
\end{aligned}
$$

\section{References}

[1] C.Y. Chia, Nonlinear Analysis of Plates, Mc Graw Hill, New York, 1980.

[2] A.H. Nayfeh, Nonlinear Interactions, Wiley, New York, 2000.

[3] F. Bloom, D. Coffin, Handbook of Thin Plate Buckling and Postbuckling, Chapman \& Hall/CRC, London, 2001.

[4] T. von Kármán, Festigkeitsprobleme im Maschinenbau, Encyklopädie der Mathematischen Wissenschaften 4 (4) (1990) 311-385 (in German).

[5] H.-N. Chu, G. Herrmann, Influence of large amplitudes on free flexural vibrations of rectangular elastic plates, Journal of Applied Mechanics 23 (1956) 532-540.

[6] J.N. Reddy, Mechanics of Laminated Composite Plates, CRC Press, London, 1997.

[7] L.H. Donnell, A new theory for the buckling of thin cylinders under axial compression and bending, Transactions of the American Society of Mechanical Engineers 56 (1934) 795-806.

[8] K. Marguerre, Zur Theorie der Gekrümmten Platte Grosser Formänderung, in: J. den Hartog, H. Peters (Eds.), Proceedings of the Fifth International Congress of Applied Mechanics, Wiley, Harvard University and MIT, September 1938, Cambridge, USA, 1939, pp. 93-101.

[9] K.M. Mushtari, K.Z. Galimov, Non-linear Theory of Thin Elastic Shells, Israel Program for Scientific Translations, 1961 (Russian ed.: 1957).

[10] A.W. Leissa, A.S. Kadi, Curvature effects on shallow shell vibrations, Journal of Sound and Vibration 16 (2) (1971) $173-187$.

[11] S. Łukasiewicz, Local Loads in Plates an Shells, Sijthoff \& Noordhoff, Alphen aan der Rijn, 1979.

[12] H.M. Berger, A new approach to the analysis of large deflections of plates, Journal of Applied Mechanics 22 (4) (1955) $465-472$.

[13] J.L. Nowinski, H. Ohnabe, On certain inconsistencies in Berger equations for large deflections of elastic plates, International Journal of Mechanical Sciences 14 (1972) 165-170.

[14] C.P. Vendhan, A study of Berger equations applied to nonlinear vibration of elastic plates, International Journal of Mechanical Sciences 17.

[15] A.H. Nayfeh, D.T. Mook, Nonlinear Oscillations, Wiley, New York, 1979.

[16] A.H. Nayfeh, P.F. Pai, Linear and Nonlinear Structural Mechanics, Wiley, New York, 2004.

[17] G. Düring, C. Josserand, S. Rica, Weak turbulence for a vibrating plate: Can one hear a Kolmogorov spectrum?, Physical Review Letters 97 (2006) 025503.

[18] A. Chaigne, C. Touzé, O. Thomas, Nonlinear vibrations and chaos in gongs and cymbals, Acoustics Science and Technology 26 (5) (2005) 403-409. 
[19] M. Ben Amar, Y. Pomeau, Crumpled paper, Proceedings of the Royal Society London A 453 (1997) 729-755.

[20] I. Kozinsky, H.W.C. Postma, I. Bargatin, M.L. Roukes, Tuning nonlinearity dynamic range and frequency of nanomechanical resonators, Applied Physics Letters 88 (2006) 263101.

[21] O. Thomas, L. Nicu, C. Ayela, C. Touzé, Buckling and non-linear vibrations of a piezoelectric stratified plate. Application to a MEMS biosensor, in: Faculdade de Engenharia, Univ. do Porto (Ed.), EUROMECH Colloquium 483: Geometrically Non-Linear Vibrations of Structures, Porto, Portugal, 2007, pp. 249-252.

[22] M.P. Païdoussis, Fluid-Structure Interactions-Vol. 1: Slender Structures and Axial Flow, Academic Press, San-Diego, 1998.

[23] A.W. Leissa, Recent studies in plate vibrations: 1981-85, part ii: complicating effects, Shock and Vibration Digest 19 (1987) 10-24.

[24] M. Sathyamoorthy, Nonlinear vibrations of plates: an update of recent research developments, Applied Mechanics Review 49 (10) (1996) S55-S62.

[25] M.S. Qatu, Recent research advances in the dynamic behavior of shells: 1989-2000, part 2: homogeneous shells, Applied Mechanics Review 55 (5) (2002) 415-434.

[26] F. Moussaoui, R. Benamar, Non-linear vibrations of shell-type structures: A review with bibliography, Journal of Sound and Vibration 255 (1) (2002) 161-184.

[27] M. Amabili, M.P. Païdoussis, Review of studies on geometrically nonlinear vibrations and dynamics of circular cylindrical shells and panels, with and without fluid-structure interaction, Applied Mechanics Review 56 (4).

[28] M. Amabili, Nonlinear Vibrations and Stability of Shells and Plates, Cambridge University Press, Cambridge, 2008.

[29] O. Thomas, C. Touzé, A. Chaigne, Asymmetric non-linear forced vibrations of free-edge circular plates, part 2: experiments, Journal of Sound and Vibration 265 (5) (2003) 1075-1101.

[30] O. Thomas, C. Touzé, É. Luminais, Non-linear vibrations of free-edge thin spherical shells: experiments on a 1:1:2 internal resonance, Nonlinear Dynamics 49 (1-2) (2006) 259-284.

[31] P.G. Ciarlet, A justification of the von Kármán equations, Archive for Rational Mechanics and Analysis 73 (1980) 349-389.

[32] D.D. Fox, A. Raoult, J.C. Simo, A justification of nonlinear properly invariant plate theories, Archive for Rational Mechanics and Analysis 124 (1993) 157-199.

[33] O. Millet, A. Hamdouni, A. Cimetière, A classification of thin plate models by asymptotic expansion of non-linear three-dimensional equilibrium equations, International Journal of Nonlinear Mechanics 36 (2001) 165-186.

[34] P.G. Ciarlet, L. Gratie, From the classical to generalized von Kármán and Marguerre-von Kármán equations, Journal of Computational and Applied Mathematics 190 (2006) 470-486.

[35] G. Friesecke, R.D. James, S. Müller, A hierarchy of plate models derived from nonlinear elasticity by gamma-convergence, Archive for Rational Mechanics and Analysis 180 (2006) 183-236.

[36] S. Bilbao, A family of conservative finite difference schemes for the dynamical von Kármán plate equations, Numerical Methods for Partial Differential Equations 24 (1) (2007) 193-216.

[37] G.J. Efstathiades, A new approach to the large-deflection vibrations of imperfect circular disks using galerkin's procedure, Journal of Sound and Vibration 16 (2) (1971) 231-253.

[38] S. Sridhar, D.T. Mook, A.H. Nayfeh, Non-linear resonances in the forced responses of plates, part I: symmetric responses of circular plates, Journal of Sound and Vibration 41 (3) (1975) 359-373.

[39] S. Sridhar, D.T. Mook, A.H. Nayfeh, Non-linear resonances in the forced responses of plates, part II: asymmetric responses of circular plates, Journal of Sound and Vibration 59 (2) (1978) 159-170.

[40] C. Touzé, O. Thomas, A. Chaigne, Asymmetric non-linear forced vibrations of free-edge circular plates, part 1: theory, Journal of Sound and Vibration 258 (4) (2002) 649-676.

[41] G. Herrmann, Influence of large amplitudes on flexural vibrations of elastic plates, Technical Report 3578, National Advisory Committee for Aeronautics (NACA), 1955.

[42] M. Géradin, D. Rixen, Mechanical Vibrations, Wiley, Chichester, 1994.

[43] O. Thomas, C. Touzé, A. Chaigne, Non-linear vibrations of free-edge thin spherical shells: modal interaction rules and 1:1:2 internal resonance, International Journal of Solids and Structures 42 (11-12) (2005) 3339-3373.

[44] A. Chaigne, C. Lambourg, Time-domain simulation of damped impacted plates, i, theory and experiments, Journal of the Acoustical Society of America 109 (4) (2001) 1422-1432.

[45] C. Touzé, O. Thomas, A. Chaigne, Hardening/softening behaviour in non-linear oscillations of structural systems using non-linear normal modes, Journal of Sound and Vibration 273 (1-2) (2004) 77-101.

[46] C. Touzé, O. Thomas, Non-linear behaviour of free-edge shallow spherical shells: effect of the geometry, International Journal of Nonlinear Mechanics 41 (5) (2006) 678-692.

[47] C. Touze, M. Amabili, O. Thomas, Reduced-order models for large-amplitude vibrations of shells including in-plane inertia, Computer Methods in Applied Mechanics and Engineering 197 (21-24) (2008) 2030-2045.

[48] E. Doedel, R. Paffenroth, A. Champneys, T. Fairgrieve, Y. Kuznetsov, B. Oldeman, B. Sandstede, X.Wang, Auto 2000: Continuation and bifurcation software for ordinary differential equations, user's guide, Technical Report, Concordia University, 2002.

[49] B. Cochelin, N. Damil, M. Potier-Ferry, Asymptotic numerical methods and Padé approximants for nonlinear elastic structures, International Journal of Numerical Methods in Engineering 37 (7) (1994) 1187-1213.

[50] B. Cochelin, N. Damil, M. Potier-Ferry, Méthode Asymptotique Numérique, Hermès, Paris, 2007 (in French).

[51] R. Arquier, Manlab: logiciel de continuation interactif (manuel utilisateur), Technical Report, Laboratoire de Mécanique et d'Acoustique, CNRS, http://www.lma.cnrs-mrs.fr/ manlab 2005 (in French). 
[52] R. Arquier, S. Bellizzi, R. Bouc, B. Cochelin, Two methods for the computation of nonlinear modes of vibrating systems at large amplitudes, Computers \& Structures 84 (2006) 1565-1576.

[53] A. Léger, B. Miara, On the multiplicity of solutions to Marguerre-von Kármán membrane equations, Journal de Mathematiques Pures et Appliques 84 (3) (2005) 357-374.

[54] M. DoCarmo, Differential Geometry of Curves and Surfaces, Prentice-Hall, Englewood Cliffs, NJ, 1976. 